\title{
Combined Reconstruction and Registration of Digital Breast Tomosynthesis
}

\author{
Guang Yang ${ }^{\dagger 1}$, John H. Hipwell ${ }^{1}$, Matthew J. Clarkson ${ }^{1,2}$, Christine Tanner ${ }^{1,3}$, \\ Thomy Mertzanidou ${ }^{1}$, Spencer Gunn ${ }^{4}$, Sebastien Ourselin ${ }^{1}$, David J. Hawkes ${ }^{1}$, \\ and Simon R. Arridge ${ }^{1}$ \\ 1 Centre for Medical Image Computing, Department of Computer Science and \\ Medical Physics, University College London (UCL), London, WC1E 6BT, UK \\ 2 Dementia Research Centre, UCL Institute Of Neurology, London, WC1N 3BG, UK \\ 3 Computer Vision Laboratory, Eidgenössische Technische Hochschule Zürich (ETH), \\ 8092, Zürich, CH \\ 4 Dexela Ltd, London N1 7EU, UK
}

\begin{abstract}
Digital breast tomosynthesis (DBT) has the potential to enhance breast cancer detection by reducing the confounding effect of superimposed tissue associated with conventional mammography. In addition the increased volumetric information should enable temporal datasets to be more accurately compared, a task that radiologists routinely apply to conventional mammograms to detect the changes associated with malignancy. In this paper we address the problem of comparing DBT data by combining reconstruction of a pair of temporal volumes with their registration. Using a simple test object, and DBT simulations from in vivo breast compressions imaged using MRI, we demonstrate that this combined reconstruction and registration approach produces improvements in both the reconstructed volumes and the estimated transformation parameters when compared to performing the tasks sequentially.
\end{abstract}

\section{Introduction}

Digital breast tomosynthesis (DBT) is an X-ray modality in which a small number of low dose X-ray images (typically between 10 and 50) are acquired over a limited angle and reconstructed into a 3D volume [1]. A key issue in the creation of DBT images is the algorithm used to perform the reconstruction. This has been a topic of substantial research with many algorithms being proposed including traditional shift-and-add (SAA) [2], filtered back-projection (FBP) [3], algebraic reconstruction technique (ART) [4], maximum-likelihood expectation maximization (MLEM) [1], and matrix inversion tomosynthesis (MITS) [5]. In addition surveys have been published comparing and contrasting the relative merits of each approach [2] [4].

Reconstructed 3D DBT images have high in-plane resolution but low out-ofplane resolution and exhibit reduced superposition of overlying tissue structures

\footnotetext{
${ }^{\dagger}$ Contact Email: G.Yang@cs.ucl.ac.uk. This work has been funded by DTI Project Digital Breast Tomosynthesis TP/7/SEN/6/1/M1577G. The authors would like to thank the UK MR Breast Screening Study (MARIBS) [6] for providing the data for this study.
} 
as compared to conventional X-ray mammography. Whilst the added depth information offered by DBT has the potential to enhance detection and diagnosis of breast cancer [7]; the greater volume of data, relative to X-ray mammography, increases the need for automated tools to aid the reading process. This is of particular importance if DBT is to be adopted in the high workload screening context.

In this paper we address the problem of comparing temporal DBT volumes via registration. This is a challenging task due to the significant artefacts associated with DBT reconstructions. These are generated by the limited field of view of the acquired images and the correspondingly large null-space in the frequency domain. Rather than registering the images after reconstruction therefore, we investigate the benefits of combining both reconstruction and registration, and test the hypothesis that the performance of each task will be enhanced as a result. We propose an iterative method of least squares optimisation for our combined reconstruction and registration scheme. This avoids the implicit assumption of missing data being equal to zero in algorithms such as in FBP.

In recent relevant research on SPECT imaging [8] Schumacher et al. present a method to combine reconstruction with motion correction using a rigid transformation. We have developed an iterative algorithm which alternates between optimising the reconstructed intensities at each time point and the affine transformation parameters between time points.

\section{Method}

Two sets of limited angle X-ray acquisitions, $\boldsymbol{y}_{\mathbf{1}} \in \mathfrak{R}^{N_{2}}$ and $\boldsymbol{y}_{\mathbf{2}} \in \mathfrak{R}^{N_{2}}$, obtained at different times, can be expressed in terms of a $3 \mathrm{D}$ volume, $\boldsymbol{x} \in \mathfrak{R}^{N_{3}}$, in two positions related by the transformation, $R$, with parameters, $\zeta_{p} \in \mathfrak{R}$, and the system matrix $A: \mathfrak{R}^{N_{3}} \mapsto \mathfrak{R}^{N_{2}}$ via

$$
\boldsymbol{y}_{1}=A \boldsymbol{x},
$$

and

$$
\boldsymbol{y}_{2}=A \boldsymbol{x}^{\dagger}=A R_{\zeta_{p}} \boldsymbol{x} .
$$

We solve equations 1 and 2 with respect to estimates $\boldsymbol{x}_{1}$ and $\boldsymbol{x}_{\boldsymbol{2}}$ of $\boldsymbol{x}$ and the registration parameters $\boldsymbol{\zeta}_{\boldsymbol{p}}$, by alternating an incomplete optimisation (i.e. $n$ iterations) of the reconstructed volumes $\boldsymbol{x}_{1}$ and $\boldsymbol{x}_{2}$ :

$$
\begin{aligned}
& \boldsymbol{x}_{\mathbf{1}}^{*}=\arg \min _{\boldsymbol{x}_{\mathbf{1}}}\left(\Phi_{R e c 1}=\frac{1}{2}\left\|A \boldsymbol{x}_{\mathbf{1}}-\boldsymbol{y}_{\mathbf{1}}\right\|_{2}^{2}\right) \\
& \boldsymbol{x}_{\mathbf{2}}^{*}=\arg \min _{\boldsymbol{x}_{\mathbf{2}}}\left(\Phi_{R e c 2}=\frac{1}{2}\left\|A \boldsymbol{x}_{\mathbf{2}}-\boldsymbol{y}_{\mathbf{2}}\right\|_{2}^{2}\right)
\end{aligned}
$$

with the registration of the current estimates $\boldsymbol{x}_{\mathbf{1}}^{*}$ and $\boldsymbol{x}_{\mathbf{2}}^{*}$ with respect to the registration parameters $\zeta_{p}$ :

$$
\zeta_{p}{ }^{*}=\arg \min _{\zeta_{p}}\left(\Phi_{R e g}=\frac{1}{2}\left\|R_{\zeta_{p}} x_{2}^{*}-x_{1}^{*}\right\|_{2}^{2}\right) .
$$


After each registration iteration (Eq. 5), and prior to the next iteration of the reconstructions (Eqs. 3 and 4), the reconstruction estimates are updated as follows (Eqs. 6 and 7).

$$
\begin{aligned}
& x_{1}=R_{\zeta_{p}} x_{2}^{*} \\
& x_{2}=x_{2}^{*}
\end{aligned}
$$

This "outer loop" of reconstruction followed by registration is repeated $m$ times. The last iteration outputs $\boldsymbol{x}_{1}=\boldsymbol{x}_{1}^{*}, \boldsymbol{x}_{\mathbf{2}}=\boldsymbol{x}_{2}^{*}$ and $R_{\zeta_{p}} \boldsymbol{x}_{\mathbf{2}}^{*}$.

The reconstruction is performed via a nonlinear conjugate gradient search engine and the registration currently via a simple hill-climbing optimisation method. The following analytical gradients are used for $\boldsymbol{x}_{1}$ and $\boldsymbol{x}_{2}$

$$
\begin{aligned}
& \Psi_{\boldsymbol{x}_{\mathbf{1}}}=A^{T}\left(A \boldsymbol{x}_{\mathbf{1}}-\boldsymbol{y}_{\mathbf{1}}\right) \\
& \Psi_{\boldsymbol{x}_{\mathbf{2}}}=A^{T}\left(A \boldsymbol{x}_{\mathbf{2}}-\boldsymbol{y}_{\mathbf{2}}\right) .
\end{aligned}
$$

The preceding combined reconstruction and registration method is summarised by

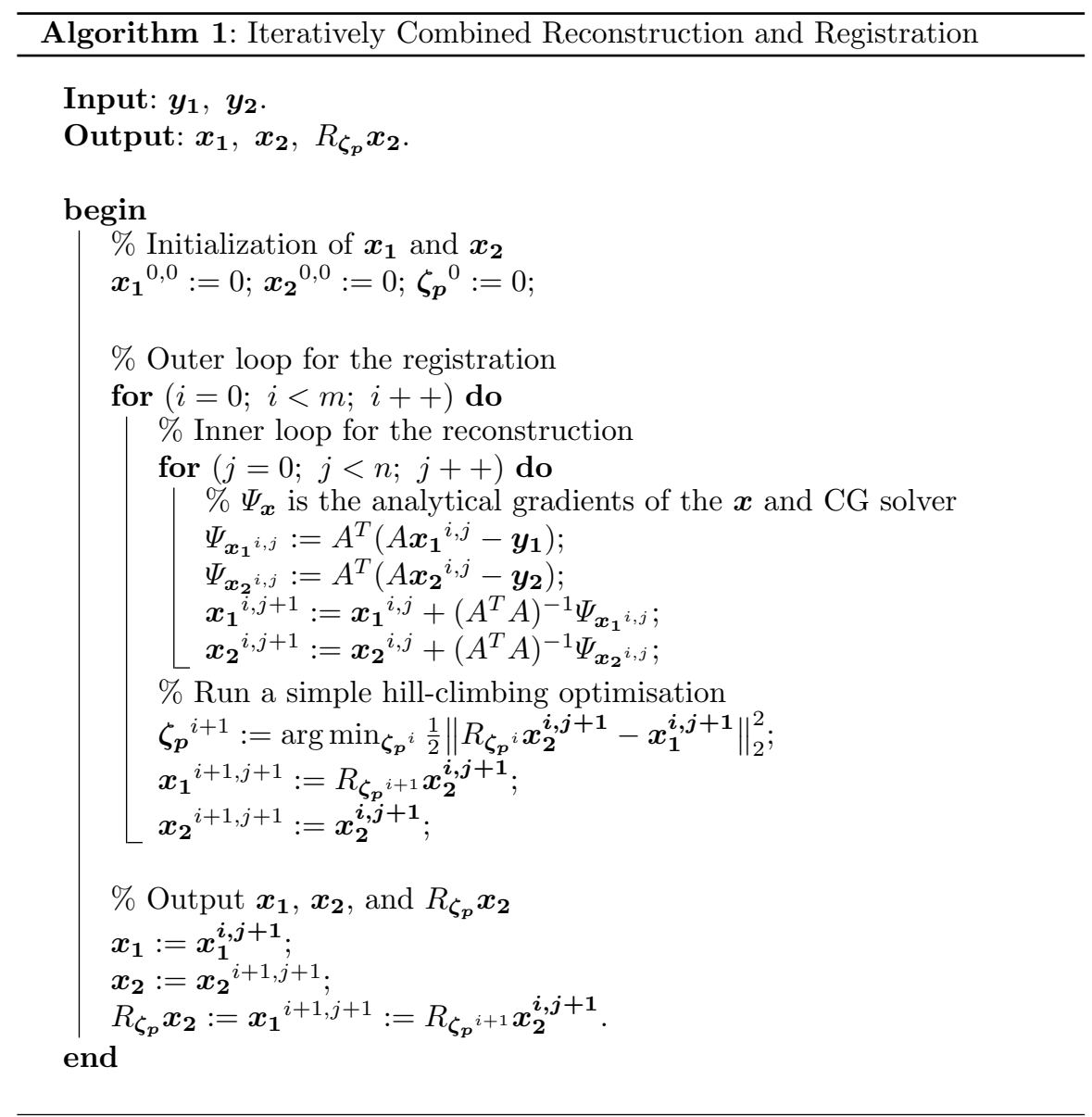




\section{Results}

In the following three experiments we compare the performance of (a) sequential reconstruction and registration, in which $n=100$ iterations of the reconstruction of projection images, $\boldsymbol{y}_{\mathbf{1}}$ and $\boldsymbol{y}_{\mathbf{2}}$, are followed by a single registration of the reconstructed volumes $\boldsymbol{x}_{\mathbf{1}}$ and $\boldsymbol{x}_{\mathbf{2}}(m=1)$, and (b) our iterative method in which $n=10$ iterations of the reconstruction are followed by a registration, and this is repeated $m=10$ times. In both cases the total reconstruction iterations are the same $(m \times n=100)$; however, there are 10 registrations in our iterative approach rather than the single registration used in the sequential method. For each pair of test volumes, $\boldsymbol{x}$ and $\boldsymbol{x}^{\dagger}, 11$ projections covering \pm 25 degrees are created to simulate the pair of temporal DBT acquisitions $\boldsymbol{y}_{\mathbf{1}}$ and $\boldsymbol{y}_{\mathbf{2}}$.

In the first experiment a $3 \mathrm{D}$ toroidal phantom image was created and rigidly transformed via parameters $R_{\zeta_{p}}$ using a translation of $T_{x, y, z}=[10,0,-20] \mathrm{mm}$ and a rotation about the $y$ axis of -30 degree (Fig. 1). As seen in Fig. 1. (f) and $(\mathrm{h})$, the iterative results are more compact and accurate than the sequential results Fig. 1. (b) and (d), and the out of plane blurring is reduced (coloured squares). The sum of squared differences (SSD) $\left\|x_{1}-x\right\|_{2}^{2}$ is decreased by an order of magnitude $\left(10^{11}\right.$ to $\left.10^{9}\right)$; however, for the iterative method this value of $4.32 \times 10^{9}$ is superior to the sequential result of $6.89 \times 10^{9}$. In the second experiment the same transformation was applied to a 3D breast MR image that obtained similar behaviour (iterative $1.25 \times 10^{8}$ vs sequential $1.42 \times 10^{8}$ decreased from $1.71 \times 10^{11}$ ) illustrated in Fig. 2. There is a black region with sharp edge at the bottom of both Fig. $2(\mathrm{~h})$ and $(\mathrm{d})$ due to the transformed image Fig. 2 (e) falling outside of the field of view. However, a better reconstruction for the missing data in Fig. 2 (f) is obtained due to our incorporation of all the X-ray acquisitions into the reconstruction of $x_{1}$.
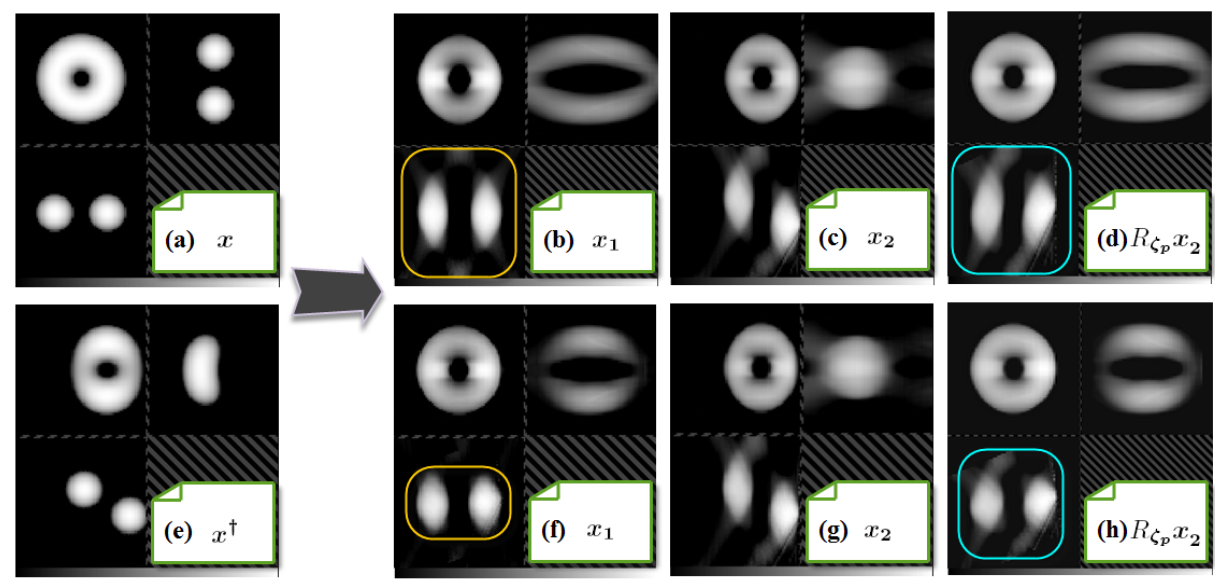

Fig. 1: (a) Original test volume $\boldsymbol{x}$; (e) Transformed test volume $\boldsymbol{x}^{\dagger}$; Sequential results (b)-(d): (b) reconstruction $\boldsymbol{x}_{1}$, (c) reconstruction $\boldsymbol{x}_{2}$, and (d) transformed reconstruction $R_{\zeta p} \boldsymbol{x}_{2}$; Iterative results $(f)-(h):(f)$ reconstruction $\boldsymbol{x}_{1},(g)$ reconstruction $\boldsymbol{x}_{2}$, and (h) transformed reconstruction $R_{\zeta p} \boldsymbol{x}_{2}$. 

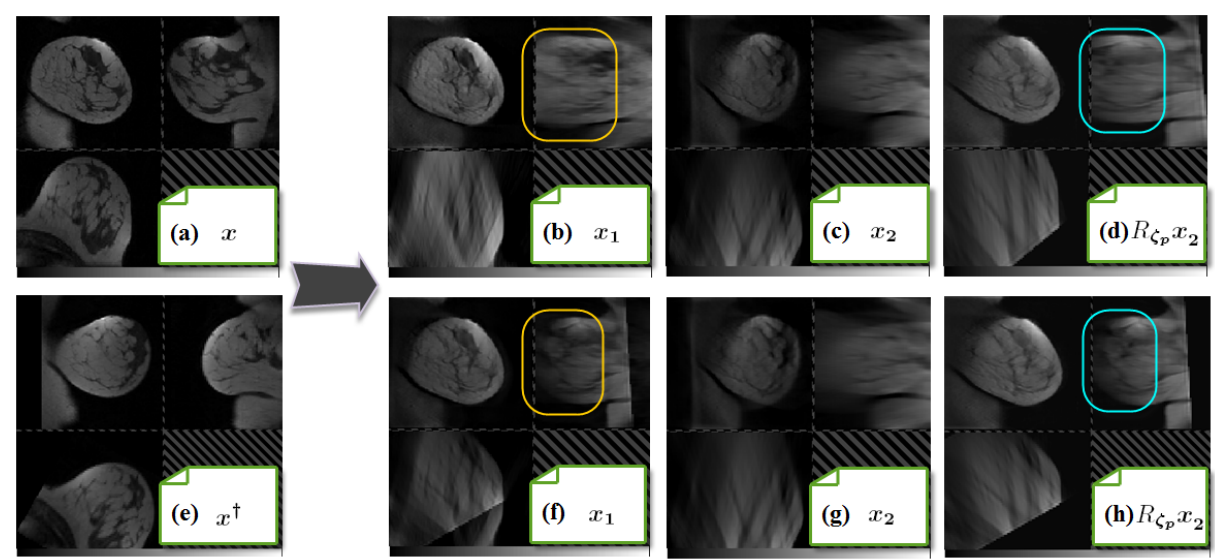

Fig. 2: As Fig. 1 but for a 3D uncompressed breast MR image.
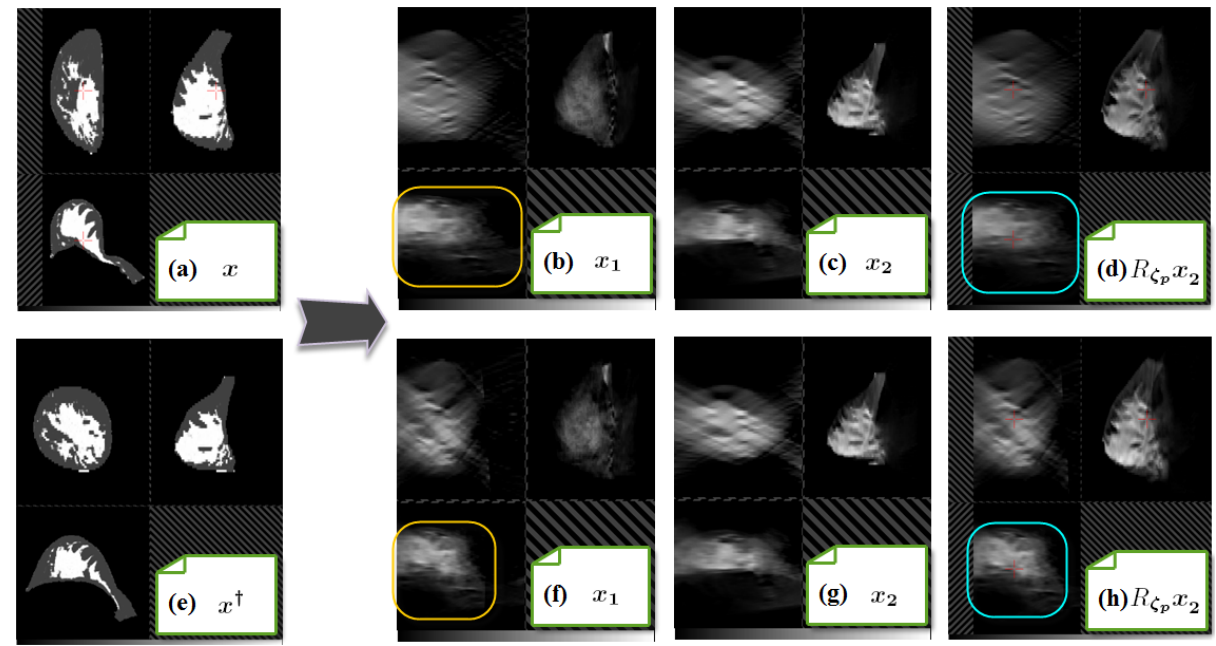

Fig. 3: As Fig. 1 but applied to in vivo MRI acquisition of a breast before and after plate compression (Images have been segmented and mapped to effective $X$-ray attenuation).

In a third experiment we tested the methods using two MRI acquisitions obtained before and after application of a lateral-to-medial plate compression of the breast (Fig. 3). The SSD between reconstruction, $\boldsymbol{x}_{\mathbf{1}}$, and the original volume, $\boldsymbol{x}$, indicates that the iterative method produces a more accurate reconstruction of the data (iterative $5.9 \times 10^{9}$ vs sequential $7.6 \times 10^{9}$ decreased from $6.91 \times 10^{11}$ ). In addition, the affine transformation model is insufficient for the compression deformation which may degrade the reconstructed results; however, measurement of the target registration error for a set of 12 user defined landmarks, indicates that the iterative method also produces a more accurate registration result (4.6mm vs $8.6 \mathrm{~mm}$, given an initial misregistration of $23.6 \mathrm{~mm}$ ). All the numerical results of the three experiments above are shown in the Table 1 below, 


\begin{tabular}{lccc}
\hline & Initial & $\begin{array}{c}\text { Combined } \\
\text { Method }\end{array}$ & $\begin{array}{c}\text { Sequential } \\
\text { Method }\end{array}$ \\
\hline Toroid SSD & $4.51 \times 10^{11}$ & $4.32 \times 10^{9}$ & $6.89 \times 10^{9}$ \\
\hline $\begin{array}{l}\text { Uncompressed } \\
\text { MRI SSD }\end{array}$ & $1.71 \times 10^{11}$ & $1.25 \times 10^{8}$ & $1.42 \times 10^{8}$ \\
\hline $\begin{array}{l}\text { Compressed } \\
\text { MRI SSD }\end{array}$ & $6.91 \times 10^{11}$ & $5.90 \times 10^{9}$ & $7.60 \times 10^{9}$ \\
\cline { 2 - 4 } $\begin{array}{l}\text { Misregistration } \\
(\mathrm{mm})\end{array}$ & 23.6 & 4.6 & 8.6 \\
\hline
\end{tabular}

Table 1: Numerical results of the three experiments. $\left(S S D=\left\|x_{1}-x\right\|_{2}^{2}\right)$

Plots of the cost function $\Phi_{R e c 1}=\left\|A \boldsymbol{x}_{\boldsymbol{1}}-\boldsymbol{y}_{\mathbf{1}}\right\|_{2}^{2}$ represented in equation 3 for both sequential and combined methods are shown in Figures 4, 5, and 6.

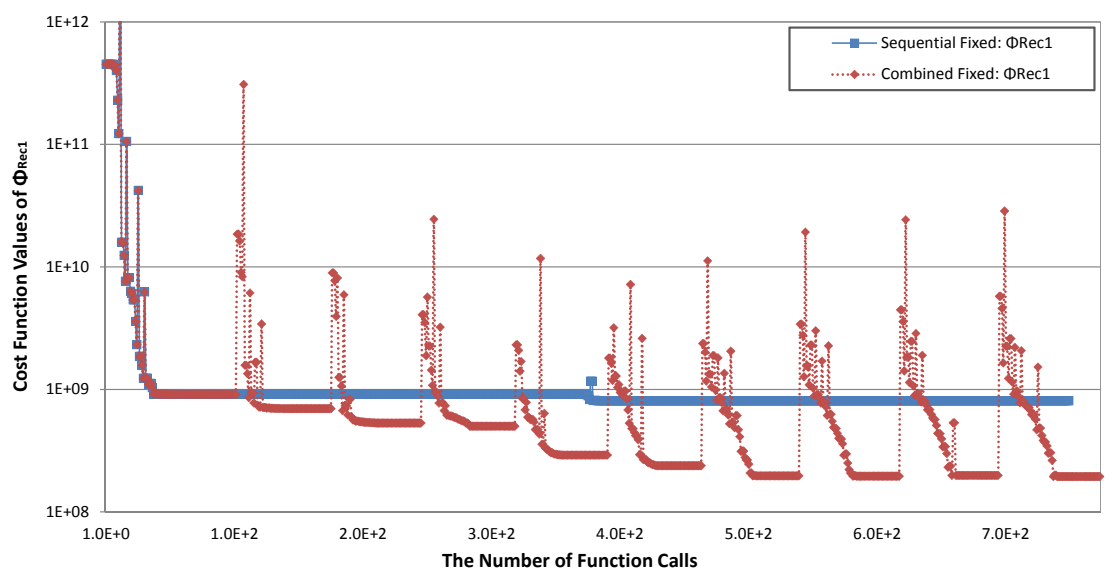

Fig. 4: Plot of the cost function $\Phi_{R e c 1}=\left\|A \boldsymbol{x}_{1}-\boldsymbol{y}_{1}\right\|_{2}^{2}$ for the $3 D$ toroid experiment.

\section{Discussion}

Our iterative method was found to produce superior results in optimised cost function value, registration accuracy and reconstructed image appearance. This is illustrated in Fig. 7. We attribute this to the fact that the iterative approach uses all the X-ray acquisition data (both $y_{1}$ and $y_{2}$ ) to reconstruct volume $x_{1}$. This leads to an improvement in the reconstruction of $x_{1}$ which in turn enables a more accurate registration to reconstructed volume $x_{2}$ to be achieved.

An implicit assumption in this approach is that there is no change in the breast (such as the growth of a tumour or due to the differences in image acquisition parameters) between the two time-points being reconstructed and registered, justifying the use of SSD as the registration similarity metric. Given this 
approach, we could envisage a subsequent step where we compare reconstruction volume $x_{1}$ with the original acquisitions, $y_{1}$ and $y_{2}$, to detect change.

The iterative method updates $x_{1}$ with the transformation of $x_{2}, R_{\boldsymbol{\zeta}_{p}} \boldsymbol{x}_{\mathbf{2}}^{*}$, after 10 iterations of the reconstruction and a single registration. This results in the 10 peaks in the cost function plot for the iterative method when compared to the smooth plot for the sequential method, Figs. 4, 5 and 6. In Fig. 5, the final cost function value of the sequential method is less than our iterative method because the MR volume has been transformed beyond the field of view $\left(\boldsymbol{x}^{\dagger}\right.$ in Fig. 2). This region is visible in the simulated projection images, $y_{1}$, however, because the $3 \mathrm{D}$ transformation is applied in the world coordinate frame. The result is that the sequential method produces a lower value of the cost function, $\Phi_{R e c 1}=\left\|A \boldsymbol{x}_{\mathbf{1}}-\boldsymbol{y}_{\mathbf{1}}\right\|_{2}^{2}$, due to greater image overlap despite the reconstruction (and registration) being less successful.

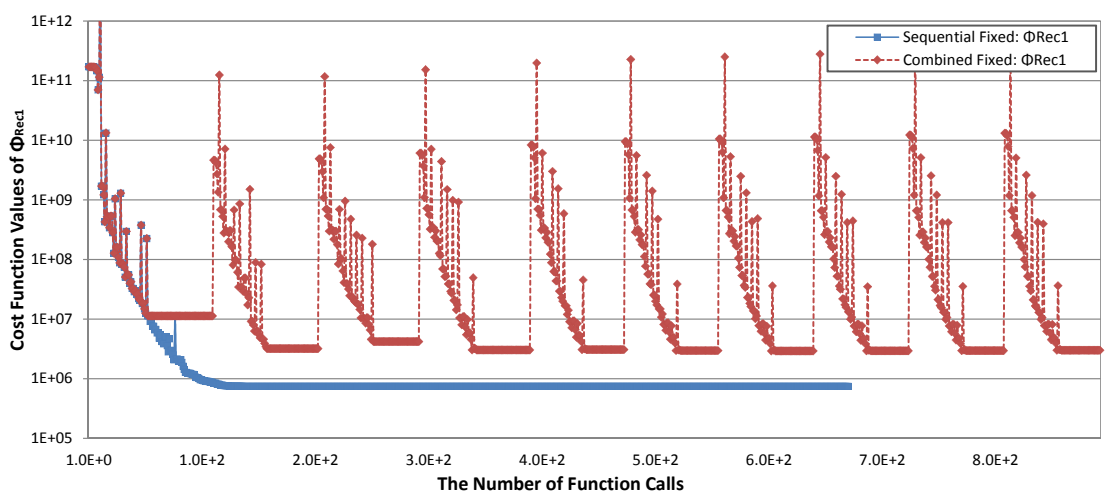

Fig. 5: As Fig. 4 but for the 3D uncompressed breast MR image.

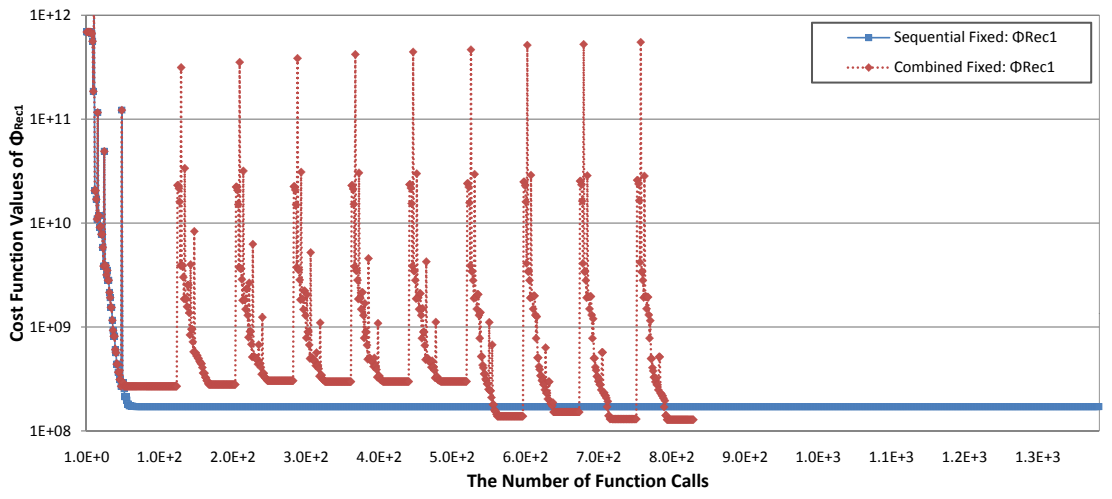

Fig. 6: As Fig. 4 but for the in vivo compressed MR experiment.

\section{Conclusion}

We have presented a method to iteratively reconstruct and register temporal DBT data sets. We have compared this approach with performing the two tasks 
sequentially and demonstrated that the former improves both the registration accuracy and the quality of the reconstructed datasets. In future work we will investigate alternative non-rigid transformations and address the issue of change in the breast tissue which may occur between time points.

\section{References}

[1] Wu, T., et al.: Tomographic Mammography Using a Limited Number of Low-dose Cone-beam Projection Images. Medical Physics 30, 365-380 (2003)

[2] Dobbins, J.T. III and Godfrey, D.J.: Digital X-ray Tomosynthesis: Current State of the Art and Clinical Potential. Phys. Med. Biol. 48, R65-R106 (2003)

[3] Lauritsch, G. and Haerer, W.: A Theoretical Framework for Filtered BackProjection in Tomosynthesis. Proceedings of SPIE 3338, 1127-1137 (1998)

[4] Zhang, Y., et al.: A Comparative Study of Limited-angle Cone-beam Reconstruction Methods for Breast Tomosynthesis. Medical physics 33, 3781-3795 (2006)

[5] Warp R.J., et al.: Applications of Matrix Inversion Tomosynthesis. Proceedings of SPIE 3977, 376-383 (2000)

[6] Leach M.O., et al.: Screening with MRI and mammography of a UK population at high familial risk of breast cancer. The Lancet 365, 1769-1778 (2005)

[7] Kopans, D.B.: Breast Imaging. $3^{\text {rd }}$ Ed., Lippincott W. \& W., Philadelphia (2007)

[8] Schumacher, H., et al.: Combined Reconstruction and Motion Correction in SPECT Imaging. IEEE Transactions on Nuclear Science 56, 73-80 (2009)
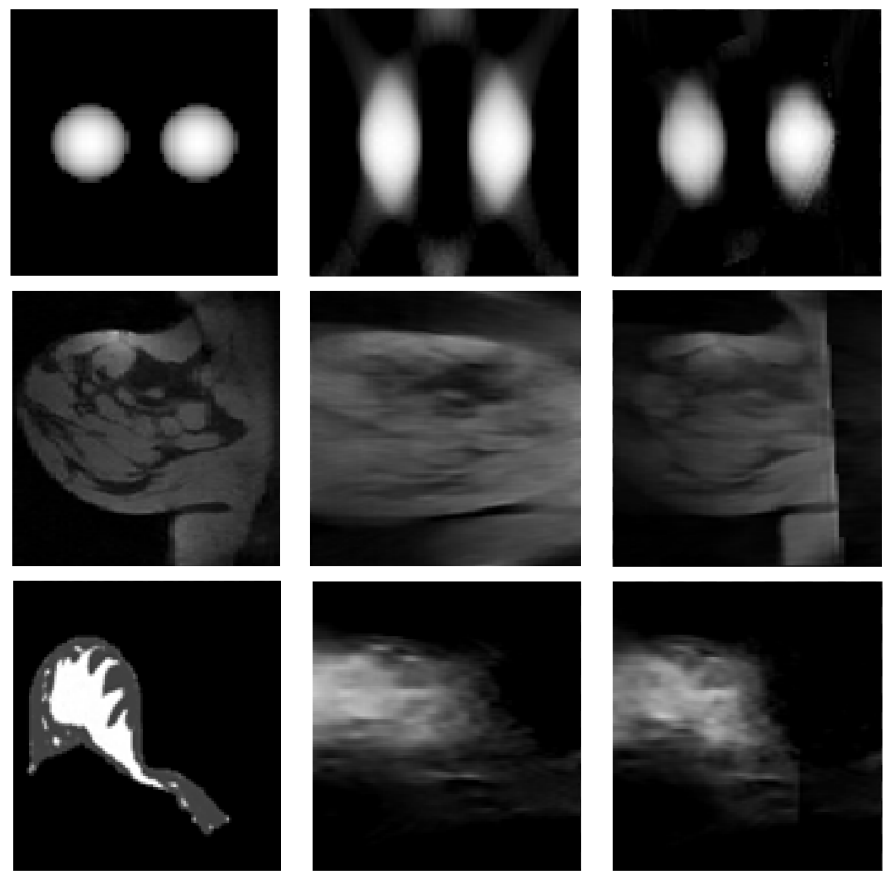

Fig. 7: Magnified results of the three tests above. (a), (b) and ( $f$ ) of figures 1, 2 and 3. Left to right: Original fixed image $x$; Results of the sequential method $x_{1}$; Results of the iterative method $x_{1}$. Only one of the out-of-plane slices has been shown accordingly. 